\title{
Feedback Optimal Control in Low-Thrust Interplanetary Trajectory Design
}

\author{
A. Owis ${ }^{\dagger}$, F. Topputo ${ }^{\ddagger}$, and F. Bernelli-Zazzera ${ }^{\ddagger}$ \\ ${ }^{\dagger}$ Dipartimento di Matematica, Università di Milano Bicocca, \\ and Astronomy Department, Cairo University \\ $\ddagger$ Dipartimento di Ingegneria Aerospaziale, Politecnico di Milano
}

\begin{abstract}
The feedback optimal control problem in low-thrust interplanetary trajectory design is studied in this paper. The problem is tackled by solving the Hamilton-Jacobi-Bellman equation via a generating function technique devised for linear systems. Instead of solving the classical optimal control problem, this technique allows us to derive closed loop control laws in the preliminary design phase.

The idea of the work consists in applying a globally diffeomorphic linearizing transformation that rearranges the original nonlinear two-body dynamics into a linear system of ordinary differential equations written in new variables. The generating function technique is then applied to this new dynamical system, the feedback optimal control is solved, and the variables are back transformed in the original ones. We circumvent in this way the problem of expanding the vector field and truncating higher-order terms because no accuracy is lost in the undertaken approach.

This technique can be applied to any planet-to-planet transfer; it has been successfully tested here for the classical Earth-Mars low-thrust transfer.
\end{abstract}

Keywords: Low-Thrust Transfers, Feedback Optimal Control, Generating Function, Hamilton-Jacobi-Bellman Equation. 


\section{Introduction and Statement of the Problem}

The usefulness of low-thrust propulsion applied to steer spacecraft has recently been demonstrated by two missions, the NASA's Deep Space-1 and the ESA's SMART-1. The high specific impulse associated to this new technology allows a sensible reduction in the propellant mass fraction needed to transfer spacecraft to a desired target; the final outcome is then a reduced mass at launch or an increased payload mass.

Although the low-thrust propulsion gives rise to advantages from the mass standpoint, the trajectory design for spacecraft equipped with these systems becomes less trivial than that associated to chemical propelled spacecraft. Indeed, the chemical propulsion is usually assumed to produce instantaneous velocity changes, while the low-thrust acts for a long time during the transfer, and needs more refined mathematical tools to be dealt with. One of these tools is the optimal control theory that is used to find the solution minimizing a certain performance index rather than just a feasible solution to the transfer problem [1].

Historically, the optimal low-thrust transfers have been tackled first with indirect and then with direct methods. The former stem from the Pontryagin's maximum principle that uses the calculus of variations [2]; the latter aim at solving the problem via a standard nonlinear programming procedure [3]. Even if it can be demonstrated that both approaches lead to the same result [4], the direct and indirect methods have different advantages and drawbacks, but in any case they require the solution of a two-point boundary value problem.

An important characteristic of the optimal trajectories designed with these classical methods is that the nominal solution is obtained in an open loop context. In other words, the optimal path, even if minimizing the prescribed performance index, is not able to respond to any perturbation that could alter the state of the spacecraft. Furthermore, if the initial conditions are slightly varied (e.g. the launch date changes), the optimal solution needs to be recomputed again. The output of the classical problem is in fact a control law expressed as a function of the time, $\mathbf{u}=\mathbf{u}(t), t_{0} \leq t \leq t_{f}$, being $t_{0}$ and $t_{f}$ the initial and final time respectively.

This work faces the optimal feedback control applied to the low-thrust interplanetary trajectory design. With this approach the optimal solutions minimize a certain performance index starting from a generic initial state $\mathbf{x}_{0}$. The outcome is a control law written in terms of the time and the initial state, $\mathbf{u}=\mathbf{u}\left(\mathbf{x}_{0}, t_{0}, t\right), t_{0} \leq t \leq t_{f}$. This represents a closed loop solution: given any initial state $\mathbf{x}_{0}$ at the time $t_{0}$, it is possible to evaluate the optimal solution starting from such state up to the final target. If for any reason the state is perturbed and assumes a new value $\mathbf{x} \prime_{0}=\mathbf{x}_{0}+\delta \mathbf{x}, t \iota_{0}=t_{0}+\delta t$, we are able to compute the new optimal solution by simply evaluating $\mathbf{u}=\mathbf{u}\left(\mathbf{x}_{0}, t \prime_{0}, t\right)$, avoiding, in this way, the solution of another two-point boundary value problem. Thus, a trajectory designed in this way has the property to respond to errors that occur during the transfer.

Another important aspect of this approach is the robustness of the solution. Once the optimal feedback control problem is solved, the solution $\mathbf{u}=\mathbf{u}\left(\mathbf{x}_{\mathbf{0}}, t_{0}, t\right)$ is available. Analyzing this function, the control law that is less sensitive to changes in the initial condition can be chosen as nominal solution. This solution is said to be robust with respect to the initial conditions.

In this paper the control function is the low-thrust per unit mass of the spacecraft and, in agreement with the principle of the solar electric propulsion, it is assumed to depend on 
the inverse of the square distance from the Sun. This type of thrust is in fact achieved by Sun-facing solar arrays that supply power to the engine. Since the power generated decreases with the inverse of the square distance from the Sun, we assume that the thrust magnitude follows the same trend.

The motion of a spacecraft is considered under the influence of the gravitational attraction of a central body, the Sun in our case, with the following assumptions: the spacecraft is subject only to the gravitational attraction of the central body along the entire trajectory; the trajectories of the planets are circular and coplanar and the motion of the spacecraft takes place in the same plane, i.e it can be described with two degrees of freedom.

The equations of motion, written in an inertial cartesian Sun-centered frame, are

$$
\ddot{\mathbf{r}}+\frac{k}{r^{3}} \mathbf{r}=\mathbf{u}
$$

where $k$ is the gravitational constant of the $\operatorname{Sun}\left(k=1.327110^{20} \mathrm{~m}^{3} / \mathrm{s}^{2}\right)$ and $\mathbf{u}$ is the acceleration given by the low thrust engine. The latter is assumed to be aligned with the Sun-spacecraft radius vector and to depend on its modulus and on the time as

$$
\mathbf{u}(r, t)=u(r, t) \frac{\mathbf{r}}{r}
$$

We now assume that the control is an explicit function of the inverse square distance from the Sun, namely

$$
u(r, t)=\frac{\varepsilon(t)}{r^{2}}
$$

where $\varepsilon(t)$ is a generic function of the time.

The dynamics is described in polar coordinates $(r, \theta)$ with dimensionless variables: the distances are normalized by the radius of the Earth's orbit, the velocities by the velocity of the Earth on its circular orbit; the times by the angular velocity of the Earth around the Sun. With these choices, the gravitational constant of the Sun turns out to be equal to one. In these coordinates the reference distance, velocity, and acceleration are $1.49610^{11} \mathrm{~m}$, $2.978510^{4} \mathrm{~m} / \mathrm{s}$, and $5.930610^{-3} \mathrm{~m} / \mathrm{s}^{2}$, respectively. The time unit turns out to be equal to 58.132 days[7].

The equations of motion in polar coordinates are

$$
\ddot{r}-r \dot{\theta}^{2}+\frac{1-\varepsilon(t)}{r^{2}}=0, \quad r \ddot{\theta}+2 \dot{r} \dot{\theta}=0 .
$$

The second of equations (4) can be rewritten as

$$
\frac{d}{d t}\left(r^{2} \dot{\theta}\right)=0
$$

meaning that the angular momentum, $h=r^{2} \dot{\theta}$, is constant during the motion even when the low-thrust acts, perturbation of the motion of the spacecraft. This conservation is due to the assumption that the control lies along the radial distance, hence its contribution to the angular momentum is zero. Moreover, the spacecraft is assumed to be initially on the 
Earth's orbit; thus, $h=r^{2} \dot{\theta}=1$ during the motion. This integral can be used to lower the order of the eqs. (4) from the fourth to the third. The new dynamical system is

$$
\ddot{r}-\frac{1}{r^{3}}+\frac{1-\varepsilon(t)}{r^{2}}=0, \quad \dot{\theta}=\frac{1}{r^{2}} .
$$

where it has been possible to decouple the dynamics of $r$ from that of $\theta$.

The system (6) can be rearranged into three first-order equations

$$
\dot{r}=v_{r}, \quad \dot{\theta}=\frac{1}{r^{2}}, \quad \dot{v}_{r}=\frac{1}{r^{3}}-\frac{1}{r^{2}}+\frac{\varepsilon(t)}{r^{2}},
$$

and rewritten in a more compact form

$$
\dot{\mathbf{x}}=\mathbf{f}(\mathbf{x})+\mathbf{u},
$$

where the vector field and the control have been purposely separated and put in a form suitable for the next section. The state, the vector field, and the control are

$$
\mathbf{x}=\left\{r, \theta, v_{r}\right\}^{T}, \quad \mathbf{f}=\left\{v_{r}, \frac{1}{r^{2}}, \frac{1}{r^{3}}-\frac{1}{r^{2}}\right\}^{T}, \quad \mathbf{u}=\left\{0,0, \frac{\varepsilon(t)}{r^{2}}\right\}^{T} .
$$

Assume now that the following performance index must be minimized

$$
J=\int_{t_{0}}^{t_{f}} \frac{\varepsilon^{2}}{r^{2}} d t=\int_{t_{0}}^{t_{f}} r^{2} u^{2} d t
$$

where $t_{0}$ and $t_{f}$ are, respectively, the initial and the final time.

The optimal control problem is stated by means of the dynamical system (8), the objective function (10), and the two-point boundary conditions

$$
\left\{\begin{array} { r l } 
{ r ( t _ { 0 } ) } & { = r _ { 0 } , } \\
{ \theta ( t _ { 0 } ) } & { = \theta _ { 0 } , } \\
{ v _ { r } ( t _ { 0 } ) } & { = 0 , }
\end{array} \quad \left\{\begin{array}{rl}
r\left(t_{f}\right) & =r_{f}, \\
\theta\left(t_{f}\right) & =\theta_{f}, \\
v_{r}\left(t_{f}\right) & =0 .
\end{array}\right.\right.
$$

\section{Solving the Optimal Feedback Control Problem for Nonlinear Dynamical Systems}

Assume that we have to minimize the following performance index

$$
J=\int_{t_{0}}^{t_{f}} \tilde{T}(\mathbf{x}, \mathbf{u}) d t
$$

subject to the nonlinear dynamics

$$
\dot{\mathbf{x}}=f(\mathbf{x})+\mathbf{u},
$$

where $f(\mathbf{x})$ is a nonlinear function, $\mathbf{x}$ is the $(n \times 1)$ state vector, and $\mathbf{u}$ is the $(n \times 1)$ control vector; the initial and final states are assumed to be given, $\mathbf{x}\left(t_{0}\right)=\mathbf{x}_{0}$ and $\mathbf{x}\left(t_{f}\right)=\mathbf{x}_{f}$. We 
search a globally diffeomorphic linearizing transformation, $\mathbf{y}=\mathbf{M}(\mathbf{x})$ and $\mathbf{u}=\alpha(\mathbf{x})+\beta(\mathbf{x}) \mathbf{v}$ such that the new state space representation for the dynamical system becomes [1]

$$
\mathbf{y}^{\prime}=A \mathbf{y}+B \mathbf{v}
$$

where $\mathbf{y} \prime=d \mathbf{y} / d \tau$, and $\tau$ is the new independent variable. $A$ and $B$ are both $(n \times n)$ constant matrices, $(M, \alpha): \mathbb{R}^{n} \rightarrow \mathbb{R}^{n}$, and $\beta$ is a $(n \times n)$ matrix depending on $\mathbf{x}$.

The derivative $\mathbf{y}$ ' can be written as

$$
\mathbf{y}^{\prime}=\frac{\partial M}{\partial \mathbf{x}} \dot{\mathbf{x}} \frac{d t}{d \tau}=\frac{\partial M}{\partial \mathbf{x}}(f(\mathbf{x})+\mathbf{u}) \frac{d t}{d \tau},
$$

while the old state and control vectors are given by the inverse transformations

$$
\mathbf{x}=\mathbf{M}^{-1}(\mathbf{y}), \quad \mathbf{u}=\alpha\left(\mathbf{M}^{-1}(\mathbf{y})\right)+\beta\left(\mathbf{M}^{-1}(\mathbf{y})\right) \mathbf{v} .
$$

The performance index can be written

$$
J=\int_{\tau_{0}}^{\tau_{f}} T(\mathbf{y}, \mathbf{v}) \frac{d t}{d \tau} d \tau
$$

The new optimal control problem is stated by eqs. (14) and (17). Once this problem is solved, $\mathbf{y}(\tau)$ and $\mathbf{v}(\tau)$ are available; $\mathbf{x}(t)$ and $\mathbf{u}(t)$ can be computed by means of the inverse transformations (16) with $[12] t=\int_{\tau_{0}}^{\tau_{f}} \frac{d t}{d \tau} d \tau$.

\subsection{Linearized Equations of Motion}

Let's consider the nonlinear dynamical system (9). The aim is now to define a globally diffeomorphic transformation that linearizes this dynamical system. If the state $\theta$ is chosen as the new independent variable, the required transformation is

$$
\mathbf{y}=\left(\begin{array}{l}
y_{1} \\
y_{2}
\end{array}\right)=\left(\begin{array}{c}
\frac{1}{r}-1 \\
-v_{r}
\end{array}\right)=M(\mathbf{x})
$$

and the time transformation is simply $\frac{d t}{d \theta}=r^{2}$, where the conservation of the angular momentum has been used. The Jacobian of the transformation (19) is

$$
\frac{\partial M}{\partial \mathbf{x}}=\left[\begin{array}{ccc}
\frac{-1}{r^{2}} & 0 & 0 \\
0 & 0 & -1
\end{array}\right],
$$

and so the derivative $\mathbf{y}$ ' can be written as

$$
\begin{aligned}
\mathbf{y}^{\prime} & =\frac{\partial M}{\partial \mathbf{x}} \dot{\mathbf{x}} \frac{d t}{d \tau}=\frac{\partial M}{\partial \mathbf{x}}(f(\mathbf{x})+\mathbf{u}) \frac{d t}{d \theta} \\
& =\left[\begin{array}{ccc}
\frac{-1}{r^{2}} & 0 & 0 \\
0 & 0 & -1
\end{array}\right]\left(\left(\begin{array}{c}
v_{r} \\
\frac{1}{r^{2}} \\
\frac{1}{r^{3}}-\frac{1}{r^{2}}
\end{array}\right)+\left(\begin{array}{c}
0 \\
0 \\
\frac{\varepsilon(t)}{r^{2}}
\end{array}\right)\right) r^{2} \\
& =A \mathbf{y}+B \mathbf{v},
\end{aligned}
$$


where

$$
A=\left[\begin{array}{cc}
0 & 1 \\
-1 & 0
\end{array}\right], \quad B=\left[\begin{array}{cc}
0 & 0 \\
0 & -1
\end{array}\right], \quad \mathbf{v}=\left(\begin{array}{c}
0 \\
\varepsilon(\theta)
\end{array}\right) .
$$

Manipulating the equation (10), the new performance index cane be defines as

$$
J=\int_{\theta_{0}}^{\theta_{f}} \mathbf{v}^{T} \mathbf{v} d \theta=\int_{\theta_{0}}^{\theta_{f}} \varepsilon^{2} d \theta .
$$

A linear state space representation, supplemented by a quadratic objective function, has been derived. The optimal feedback control problem can be now solved by means of the standard methods available for these systems.

\subsection{Linear Quadratic Controller}

Consider the problem of minimizing the following performance index

$$
J=\frac{1}{2} \int_{t_{0}}^{t_{f}}\left(\mathbf{y}^{T} Q \mathbf{y}+\mathbf{v}^{T} R \mathbf{v}\right) d t
$$

subject to the linear dynamics

$$
\dot{\mathbf{y}}=A \mathbf{y}+B \mathbf{v},
$$

and with the given initial and final conditions

$$
\mathbf{y}\left(t_{0}\right)=\mathbf{y}_{0}, \quad \mathbf{y}\left(t_{f}\right)=\mathbf{y}_{f}
$$

According to the classical theory, the Hamiltonian of the optimal control problem (23)$(25)$ is

$$
H=\frac{1}{2}\left(\mathbf{y}^{T} Q \mathbf{y}+\mathbf{v}^{T} R \mathbf{v}\right)+\boldsymbol{\lambda}^{T}(A \mathbf{y}+B \mathbf{v}),
$$

where the set of Lagrangian multipliers $\boldsymbol{\lambda}$ has been introduced.

From the optimality condition

$$
\frac{\partial H}{\partial \mathbf{v}}=0
$$

it is possible to get an explicit expression for the control in terms of the Lagrangian multipliers

$$
\mathbf{v}=-R^{-1} B^{T} \boldsymbol{\lambda} .
$$

Substituting the expression of $\mathbf{v}$ given by equation (28), the Hamiltonian (26) turns out to be

$$
H=\frac{1}{2}\left(\begin{array}{c}
\mathbf{y} \\
\boldsymbol{\lambda}
\end{array}\right)^{T}\left[\begin{array}{cc}
Q & A^{T} \\
A & -B R^{-1} B^{T}
\end{array}\right]\left(\begin{array}{c}
\mathbf{y} \\
\boldsymbol{\lambda}
\end{array}\right),
$$

while the dynamics of the system and that of the Lagrange multipliers reduces to

$$
\left(\begin{array}{l}
\dot{\mathbf{y}} \\
\dot{\boldsymbol{\lambda}}
\end{array}\right)=\left[\begin{array}{cc}
A & -B R^{-1} B^{T} \\
-Q & -A^{T}
\end{array}\right]\left(\begin{array}{l}
\mathbf{y} \\
\boldsymbol{\lambda}
\end{array}\right) .
$$




\subsection{The Generating Function Technique}

Suppose now that we have a generating function $F_{2}\left(\mathbf{y}, \boldsymbol{\lambda}_{0}, t, t_{0}\right)$ for the transformation between a fixed state $\left(\mathbf{y}_{0}, \boldsymbol{\lambda}_{0}, t_{0}\right)$ and a moving state $(\mathbf{y}, \boldsymbol{\lambda}, t)$. This transformation is canonical because it generates the identity transformation at $t=t_{0}$ and preserves the area in the phase space. Since the Hamiltonian is quadratic, $F_{2}$ can be put in a quadratic form as follows [8]

$$
F_{2}\left(\mathbf{y}, \boldsymbol{\lambda}_{0}, t, t_{0}\right)=\frac{1}{2}\left(\begin{array}{c}
\mathbf{y} \\
\boldsymbol{\lambda}_{0}
\end{array}\right)^{T}\left[\begin{array}{cc}
F_{y y}\left(t, t_{0}\right) & F_{y \lambda_{0}}\left(t, t_{0}\right) \\
F_{\lambda_{0} y}\left(t, t_{0}\right) & F_{\lambda_{0} \lambda_{0}}\left(t, t_{0}\right)
\end{array}\right]\left(\begin{array}{c}
\mathbf{y} \\
\boldsymbol{\lambda}_{0}
\end{array}\right),
$$

which can be used to find the unknown boundary conditions using the given ones. From the properties of $F_{2}$ we have

$$
\boldsymbol{\lambda}=\frac{\partial F_{2}}{\partial \mathbf{y}}=\left(\begin{array}{ll}
F_{y y} & F_{y \lambda_{0}}
\end{array}\right)\left(\begin{array}{c}
\mathbf{y} \\
\boldsymbol{\lambda}_{0}
\end{array}\right) .
$$

The Hamiltonian (29) can be expressed as a function of $\left(\mathbf{y}, \boldsymbol{\lambda}_{0}\right)$ by using equation (32)

$$
H=\frac{1}{2}\left(\begin{array}{c}
\mathbf{y} \\
\boldsymbol{\lambda}_{0}
\end{array}\right)^{T}\left[\begin{array}{cc}
I & F_{y y} \\
0 & F_{\lambda_{0} y}
\end{array}\right]\left[\begin{array}{cc}
Q & A^{T} \\
A & -B R^{-1} B^{T}
\end{array}\right]\left[\begin{array}{cc}
I & 0 \\
F_{y y} & F_{y \lambda_{0}}
\end{array}\right]\left(\begin{array}{c}
\mathbf{y} \\
\boldsymbol{\lambda}_{0}
\end{array}\right) .
$$

Since the Hamiltonian at the fixed state can be taken zero without any loss of generality, then the Hamiltonian of the moving state and the generating function satify the Hamilton-Jacobi PDE

$$
\begin{aligned}
0= & \left(\begin{array}{c}
\mathbf{y} \\
\boldsymbol{\lambda}_{0}
\end{array}\right)^{T}\left[\begin{array}{cc}
\dot{F}_{y y} & \dot{F}_{y \lambda_{0}} \\
\dot{F}_{\lambda_{0} y} & \dot{F}_{\lambda_{0} \lambda_{0}}
\end{array}\right]+ \\
& {\left[\begin{array}{cc}
I & F_{y y} \\
0 & F_{\lambda_{0} y}
\end{array}\right]\left[\begin{array}{cc}
Q & A^{T} \\
A & -B R^{-1} B^{T}
\end{array}\right]\left[\begin{array}{cc}
I & 0 \\
F_{y y} & F_{y \lambda_{0}}
\end{array}\right]\left(\begin{array}{c}
\mathbf{y} \\
\boldsymbol{\lambda}_{0}
\end{array}\right), }
\end{aligned}
$$

whose sub-matrix components provide the following set of matrix ODEs for (Riccati equations) $F_{y y}\left(t, t_{0}\right), F_{y \lambda_{0}}\left(t, t_{0}\right)=F_{\lambda_{0} y}^{T}\left(t, t_{0}\right)$, and $F_{\lambda_{0} \lambda_{0}}\left(t, t_{0}\right)$

$$
\begin{aligned}
\dot{F}_{y y}+Q+F_{y y} A+A^{T} F_{y y}-F_{y y} B R^{-1} B^{T} F_{y y} & =0 \\
\dot{F}_{y \lambda_{0}}+A^{T} F_{y \lambda_{0}}-F_{y y} B R^{-1} B^{T} F_{y \lambda_{0}} & =0, \\
\dot{F}_{\lambda_{0} \lambda_{0}}-F_{\lambda_{0} y} B R^{-1} B^{T} F_{y \lambda_{0}} & =0 .
\end{aligned}
$$

The initial conditions which verify the identity transformation at $t=t_{0}$ are

$$
\begin{aligned}
F_{y y}\left(t_{0}, t_{0}\right) & =0_{n \times n}, \\
F_{y \lambda_{0}}\left(t_{0}, t_{0}\right) & =I_{n \times n}, \\
F_{\lambda_{0} \lambda_{0}}\left(t_{0}, t_{0}\right) & =0_{n \times n} .
\end{aligned}
$$

The problem is now the solution of the set of ODE (35) supplemented by the initial conditions (36). Indeed, since

$$
\mathbf{y}_{0}=\frac{\partial F_{2}}{\partial \boldsymbol{\lambda}_{0}}=F_{\lambda_{0} y} \mathbf{y}_{f}+F_{\lambda_{0} \lambda_{0}} \boldsymbol{\lambda}_{0}
$$

the initial Lagrange multiplier can be evaluated through

$$
\boldsymbol{\lambda}_{0}=F_{\lambda_{0} \lambda_{0}}^{-1}\left(t_{f}, t_{0}\right)\left(\mathbf{y}_{0}-F_{\lambda_{0} y}\left(t_{f}, t_{0}\right) \mathbf{y}_{f}\right) .
$$




\section{Optimal Feedback Low-Thrust Transfers}

The optimal low-thurst planet-to-planet transfer has been stated through equations (8)-(11). The linearizing transformation has been used to derive the linear state space representation (20) with the quadratic objective function (22). This is the case of a linear quadratic controller, and the generating function technique can be applied to solve this problem. Once the linear problem is solved, the variables can be transformed back to the original ones and the optimal feedback low-thrust interplanetary transfer can be evaluated.

For the problem at hand, it turns out that $Q=0_{2 \times 2}$, and $R=2$; moreover, the initial and final states are assumed to be given by conditions (11). In the linearized variables, the initial conditions become

$$
\mathbf{y}_{\mathbf{0}}=\left(\begin{array}{c}
1 / r_{0}-1 \\
v_{r 0}
\end{array}\right), \quad \mathbf{y}_{\mathbf{f}}=\left(\begin{array}{c}
1 / r_{f}-1 \\
v_{r f}
\end{array}\right) .
$$

and, for the Earth-Mars transfer case, with the normalized variables introduced in section 1 , these conditions are $\mathbf{y}_{0}=(0,0)^{T}$, and $\mathbf{y}_{f}=(-0.3333,0)^{T}$, where $r_{0}=1, r_{f}=1.5$. In agreement with the essence of the feedback control, the initial value of the independent variable, $\theta_{0}$, is left free, while its final value has been assumed $\theta_{f}=\pi$.

Substituting $A, B, Q$, and $R$ in equation (30), and replacing the dot with the prime gives

$$
\left(\begin{array}{l}
\mathbf{y}^{\prime} \\
\boldsymbol{\lambda}^{\prime}
\end{array}\right)=\left[\begin{array}{cccc}
0 & 1 & 0 & 0 \\
-1 & 0 & 0 & -1 / 2 \\
0 & 0 & 0 & 1 \\
0 & 0 & -1 & 0
\end{array}\right]\left(\begin{array}{l}
\mathbf{y} \\
\boldsymbol{\lambda}
\end{array}\right) .
$$

Furthermore, substituting $A, B, Q$, and $R$ in equation (35) and applying the initial conditions (36), the solution can be found analytically

$$
\begin{aligned}
& F_{y y}=\left[\begin{array}{ll}
0 & 0 \\
0 & 0
\end{array}\right], \\
& F_{y \lambda}=F_{\lambda y}^{T}=\left[\begin{array}{cc}
\cos \theta & \sin \theta \\
-\sin \theta & \cos \theta
\end{array}\right], \\
& F_{\lambda \lambda}=\left[\begin{array}{cc}
-1 / 2 \sin (2 \theta)+\theta / 4 & 1 / 4 \sin ^{2} \theta \\
1 / 4 \sin ^{2} \theta & 1 / 2 \sin (2 \theta)+\theta / 4
\end{array}\right],
\end{aligned}
$$

From equation (38) we get

$$
\begin{aligned}
& \lambda_{10}=\alpha_{1}\left(y_{10}, y_{20}, \theta_{0}\right) \beta\left(\theta_{0}\right)^{-1} \cos \left(\theta-\theta_{0}\right)+\alpha_{2}\left(y_{10}, y_{20}, \theta_{0}\right) \beta\left(\theta_{0}\right)^{-1} \sin \left(\theta-\theta_{0}\right), \\
& \lambda_{20}=-\alpha_{1}\left(y_{10}, y_{20}, \theta_{0}\right) \beta\left(\theta_{0}\right)^{-1} \sin \left(\theta-\theta_{0}\right)+\alpha_{2}\left(y_{10}, y_{20}, \theta_{0}\right) \beta\left(\theta_{0}\right)^{-1} \cos \left(\theta-\theta_{0}\right),
\end{aligned}
$$

with

$$
\begin{aligned}
\alpha_{1}\left(y_{10}, y_{20}, \theta_{0}\right)= & -4 y_{10} \cos \theta_{0} \sin \theta_{0}+1.3332 \theta_{0} \cos \theta_{0}-1.3332 \pi \cos \theta_{0}+ \\
& 4 \pi y_{10}+1.3332 \sin \theta_{0}+4 y_{20}-4 y_{20} \cos \left(\theta_{0}\right)^{2}-4 y_{10} \theta_{0}, \\
\alpha_{2}\left(y_{10}, y_{20}, \theta_{0}\right)= & 4 y_{20} \sin \theta_{0} \cos \theta_{0}-4 y_{20} \theta_{0}+4 \pi y_{20}+ \\
& 1.3332 \pi \sin \theta_{0}+4 y_{10}-4 y_{10} \cos \left(\theta_{0}\right)^{2}-1.3332 \theta_{0} \sin \theta_{0}, \\
\beta\left(\theta_{0}\right)= & 8.8696+\theta_{0}^{2}+\cos \left(\theta_{0}\right)^{2}-2 \pi \theta_{0},
\end{aligned}
$$


Finally, integrating equations (40), the feedback solution for the linearized problem can be achieved

$$
\begin{aligned}
y_{1}= & \alpha_{1}\left(y_{10}, y_{20}, \theta_{0}\right) \beta\left(\theta_{0}\right)^{-1}\left(y_{10} \cos \left(\theta-\theta_{0}\right)+y_{20} \sin \left(\theta-\theta_{0}\right)+\right. \\
& \left(-\theta / 4 \cos \left(\theta-\theta_{0}\right)+\theta_{0} / 4 \cos \left(\theta-\theta_{0}\right)+1 / 4 \sin \left(\theta-\theta_{0}\right)\right)+ \\
& \alpha_{2}\left(y_{10}, y_{20}, \theta_{0}\right) \beta\left(\theta_{0}\right)^{-1}\left(\sin \left(\theta-\theta_{0}\right)+\theta_{0} / 4 \sin \left(\theta-\theta_{0}\right)\right), \\
y_{2}= & -\alpha_{1}\left(y_{10}, y_{20}, \theta_{0}\right) \beta\left(\theta_{0}\right)^{-1}\left(y_{10} \sin \left(\theta-\theta_{0}\right)+y_{20} \cos \left(\theta-\theta_{0}\right)+\right. \\
& \left(\theta / 4 \sin \left(\theta-\theta_{0}\right)-\theta_{0} / 4 \sin \left(\theta-\theta_{0}\right)\right)+\alpha_{2}\left(y_{10}, y_{20}, \theta_{0}\right) \beta\left(\theta_{0}\right)^{-1} \\
& \left(-\theta / 4 \cos \left(\theta-\theta_{0}\right)+\theta_{0} / 4 \sin \left(\theta-\theta_{0}\right)-1 / 4 \sin \left(\theta-\theta_{0}\right)\right) .
\end{aligned}
$$

A nominal solution is chosen having $\theta_{0}=0, r_{0}=1, v_{r 0}=0$, and $\theta_{f}=\pi, r_{f}=1.5$, $v_{r f}=0$. This solution corresponds to the classic Earth-Mars transfer. In figures 1, 2, and 3 , the initial conditions of the nominal solution have been perturbed in terms of $r_{0}, v_{r 0}$, and $\theta_{0}$, respectively. The new optimal feedback solution corresponding to these perturbed initial conditions is simply obtained by evaluating the solution (46).

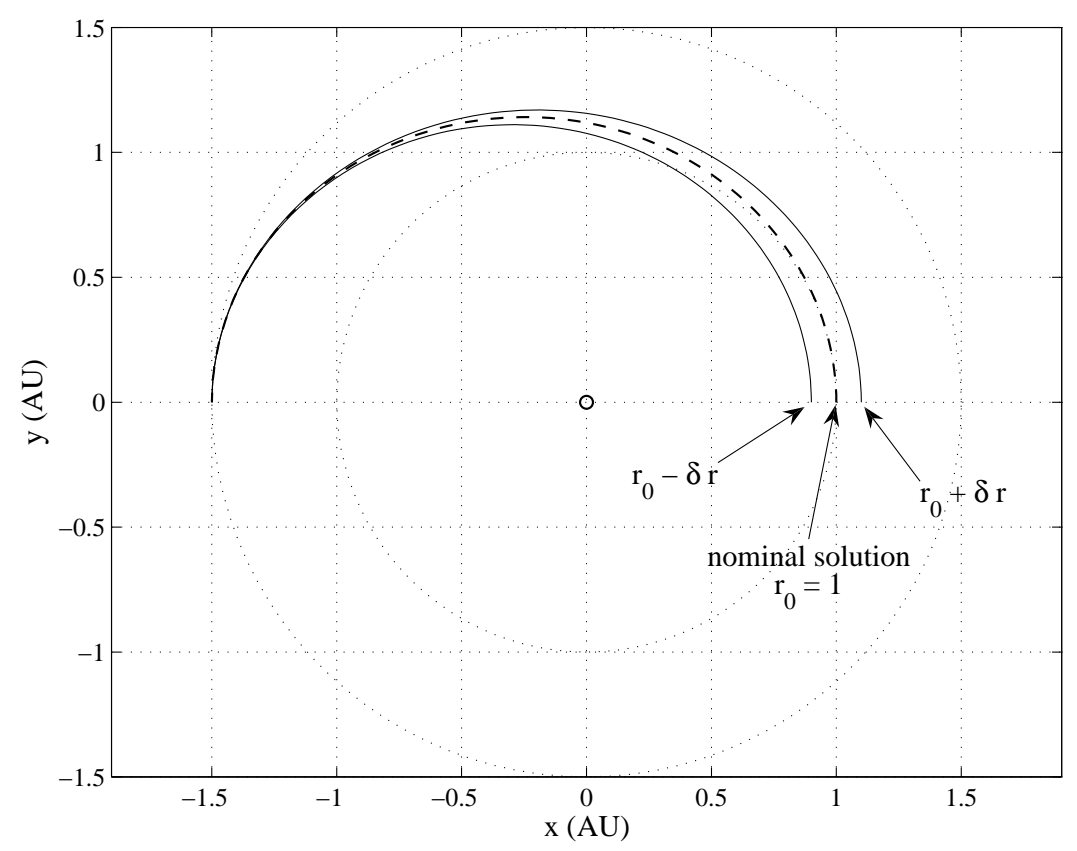

Figure 1: Nominal solution $\left(\theta_{0}=0, r_{0}=1, v_{r 0}=0\right)$ and two perturbed solutions with initial conditions $r \prime_{0}=r_{0} \pm \delta r_{0}, \delta r_{0}=0.1$ adimensional units. 


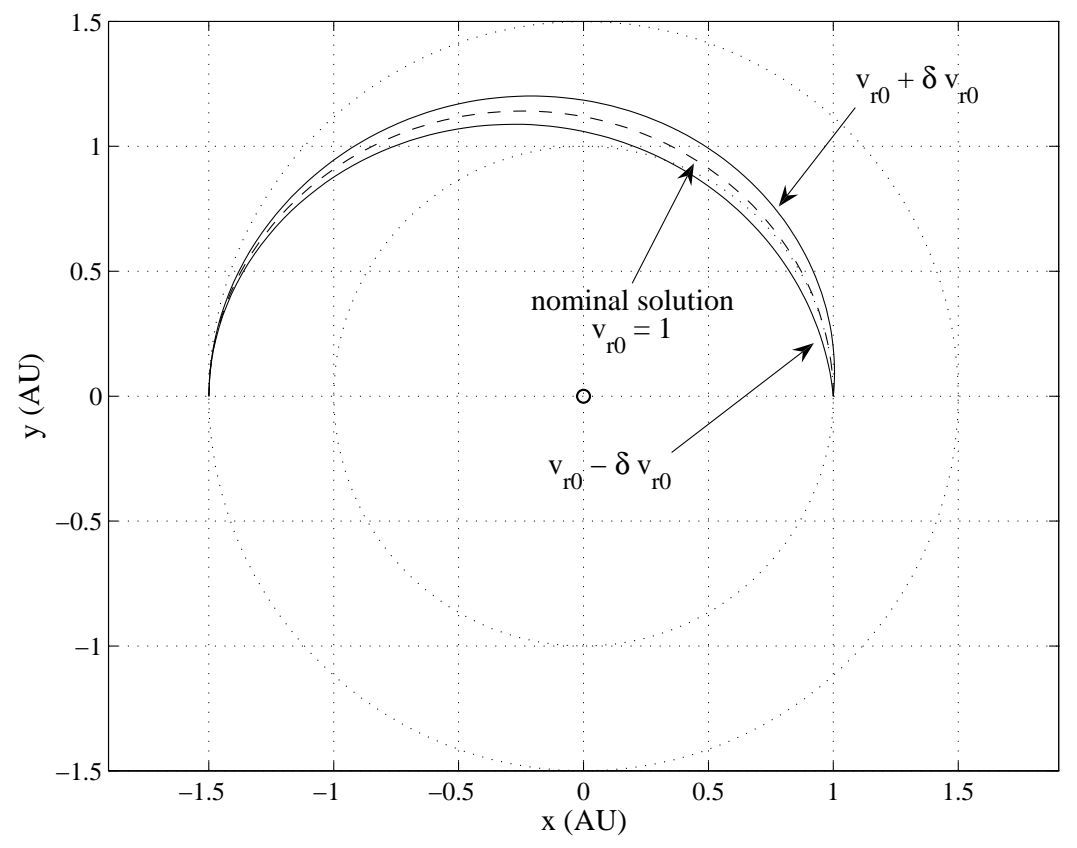

Figure 2: Nominal solution $\left(\theta_{0}=0, r_{0}=1, v_{r 0}=0\right)$ and two perturbed solutions with initial conditions $v_{r 0}^{\prime}=v_{r 0} \pm \delta v_{r 0}, \delta v_{r 0}=0.1$ adimensional units.

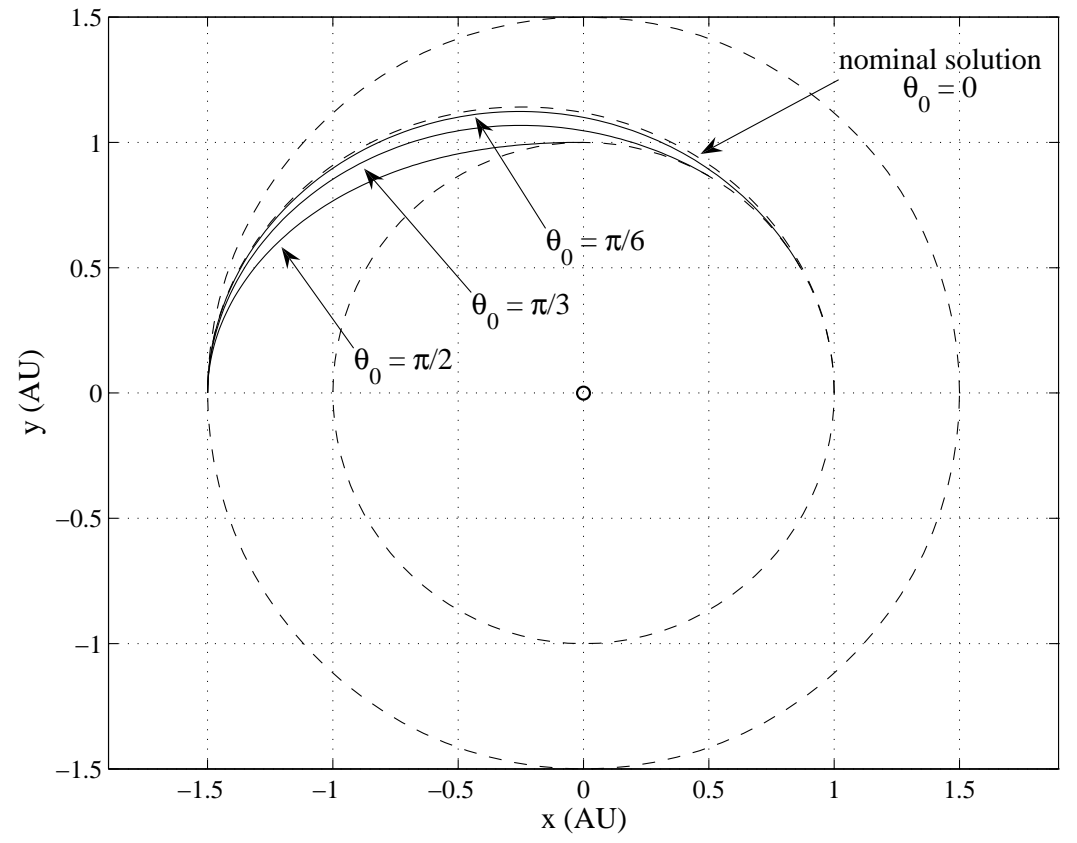

Figure 3: Nominal solution $\left(\theta_{0}=0, r_{0}=1, v_{r 0}=0\right)$ and three perturbed solutions with $\theta \prime_{0}=\theta_{0}+i \delta \theta$, $\delta \theta=\pi / 6, i=1,2,3$. 


\section{Conclusions}

The optimal feedback control problem has been solved for low-thrust orbital transfers between two circular coplanar orbits. The nonlinear problem has been transformed into a classic linear quadratic regulator problem by means of a diffeomorphic transformation. In these new variables, the dynamics is represented by a linear system while the objective function is generally a quadratic form of the states and the controls. The accuracy is totally preserved in this process since the transformation does not represent a Taylor linearization of the original nonlinear vector field. Once the problem is stated in these new variables, the optimal feedback control problem is solved by virtue of the generating function technique. The solution to this problem is back transformed into the original variables and so the optimal solution to the original problem is available in terms of generic initial and final conditions.

The effectiveness of the solution found has been tested numerically by taking perturbed initial conditions around the nominal solution. Ongoing work is focused on the generalization of the control direction along both the radial and tangential directions.

\section{References}

[1] A.E. Bryson and Y.C. Ho - Applied Optimal Control - Hemisphere, Washington, 1975

[2] L.S. Pontryagin, V.G. Boltyanskii, R.V. Gamkrelidze, and E.F. Mishchenko - The Mathematical Theory of Optimal Processes - John Wiley \& Sons, New York, 1962

[3] J.T. Betts - Practical Methods for Optimal Control using Nonlinear Programming SIAM, 2000

[4] P.J. Enright and B.A. Conway - Discrete Approximations to Optimal Trajectories Using Direct Transcription and Nonlinear Programming - Journal of Guidance, Control, and Dynamics, 15, pp. 994-1002, 1992

[5] A.J. Traskand, W.J. Mason, and V.L. Coverstone - Optimal Interplanetary Trajectories Using Constant Radial Thrust and Gravity Assist - Jounal of Guidance, Control, and Dynamics, 27, pp. 503-506, 2004

[6] A. Miele, T.Wang, and P.N. Williams - Optimal Interplanetary Orbit Transferes Via Electrical Engine - Journal of Optimization Theory and Applications, 127, pp. 605-625, 2005

[7] C.D. Murray and S.F. Dermott - Solar System Dynamics Cambrige University Press, 1999

[8] C. Park and D.J. Scheers - Solutions of Optimal Feedback Control Problems with General Boundary Conditions Using Hamiltonian Dynamics and Generating Functions American Control Conference, Boston, Massachusetts, 2004

[9] H. Yamakawa - Optimal Radially Accelerated Interplanetary Trajectories - Journal of Spacecraft and Rocket, 43, pp. 116-120, 2006 
[10] S.C.Beeler and H.T. Banks - Feedback Control Methodology for Nonlinear Systems Journal of Optimization Theory and Applications, 107, pp. 1-33, 2000

[11] S.K. Agrawal and N. Faiz - Optimization of a Class of Nonlinear Dynamic Systems: New Efficient Method without Lagrange Multipliers - Journal of Optimization Theory and Applications, 97, pp. 11-28, 1998

[12] T.Carter and M. Humi - Fuel Optimal Rendezvous Near a Point in General Keplerian Orbit - Jounal of Guidance, Control, and Dynamics, 10, pp. 567-573, 1987 